\title{
Projets pour la formation postgraduée: les idées ne manquent pas
}

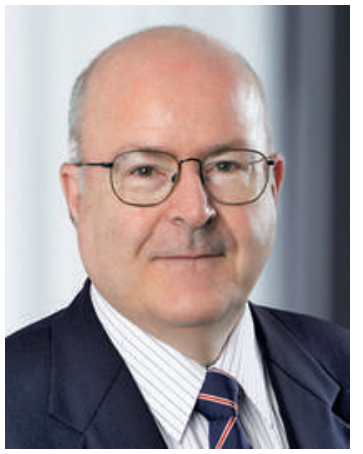

A l'origine, il y a une inquiétude et une idée. Une inquiétude, tout d'abord, concernant les conditions-cadres de la formation postgraduée des médecins, telles qu'elles sont actuellement vécues dans les hôpitaux. Lors de visites et d'autres rencontres avec les responsables de la formation, nous sommes en effet régulièrement confrontés à l'insatisfaction, l'irritation voire la résignation. Certes, l'enthousiasme et le devoir de transmettre les connaissances et les aptitudes médicales à la nouvelle génération sont toujours intacts. Cependant, dans de nombreux établissements, la réalité du quotidien hospitalier et la pression qui l'accompagne, le manque de ressources humaines et financières ainsi que les contraintes administratives entament fortement cet enthousiasme. Nombre de formateurs ont le sentiment de devoir tout faire afin de trouver le temps et l'énergie nécessaires pour transmettre activement leurs compétences dans un contexte à forte dominante économique défavorable à la formation.

\section{Les responsables de la formation subissent toujours plus la pression du temps et des coûts.}

Est-ce vraiment ce que nous voulons? L'idée de lancer un appel à projets a germé lors de notre réflexion sur les mesures susceptibles de stimuler l'enseignement. Nous avions la chance de disposer d'un budget permettant de consacrer env. 100000 francs à la promotion de projets visant à aider ou à décharger les formateurs. En octobre 2013, nous avons lancé notre appel à projets en optant non pas pour une définition précise des travaux attendus mais en encourageant délibérément l'envoi de propositions les plus diverses, que nous avons attendues avec impatience.

A notre grande satisfaction, notre appel à projets a rencontré un large écho. Pas moins de 62 travaux nous ont été soumis, la plupart d'une qualité et d'une originalité hors du commun. Si nous avons bien évidemment aussi reçu quel- ques projets plutôt fantaisistes, qui visaient simplement à récolter un financement pour la formation au quotidien, les concepts novateurs et créatifs l'emportaient clairement. Sur le plan du contenu, les projets portaient aussi bien sur des outils d'apprentissage interactifs, des méthodes hybrides, des instruments de simulation que sur la création de «skill labs» et de banques de données, des applications pour évaluer le quotidien clinique, des cours standardisés pour la mise au courant de nouveaux collaborateurs, des offres de formation destinées aux formateurs ou encore des scénarios pour ate-

\section{Avec 62 projets soumis, l'appel à projets de I'ISFM a rencontré un large écho.}

liers. C'est donc une tâche aussi enrichissante que difficile qui attendait le jury, chargé de faire un choix parmi un grand nombre de projets prometteurs tout en respectant le budget. La possibilité de mettre en œuvre le projet en dehors du lieu dans lequel il a été conçu a été l'un des critères déterminants dans le choix cornélien du jury. Suite à une sélection en deux phases, quatre projets ont finalement été retenus et recevront un soutien financier. Ces derniers portent sur la création d'une plateforme d'apprentissage en radiologie, la réalisation d'une banque de données en cytopathologie par les médecins en formation, le développement d'un système d'entraînement en chirurgie mini-invasive et la mise sur pied d'un cours de simulation en ventilation mécanique pour la médecine intensive.

Et à présent? Nous sommes convaincus que les projets primés pourront être mis en œuvre avec succès y compris sur le long terme et qu'ils contribueront à promouvoir la formation postgraduée bien au-delà de l'établissement dans lequel ils ont été conçus. Nous savons désormais que les idées visant à promouvoir la formation postgraduée ne manquent pas et nous avons la ferme intention de les soutenir dans le cadre de nos possibilités en répétant cette expérience.

Dr Werner Bauer, président de l'Institut suisse pour la formation médicale postgraduée et continue (ISFM) 